\title{
Mobile Photo Browsing with Pipelines and Spatial Cues
}

\author{
Tero Hakala, Juha Lehikoinen, Hannu Korhonen, and Aino Ahtinen \\ Nokia Research Center, \\ P.O. Box 100, \\ 33721 Tampere, Finland \\ \{tero.hakala, juha.lehikoinen, hannu.j.korhonen, \\ aino.ahtinen\} @nokia.com
}

\begin{abstract}
Local memory in mobile devices increases rapidly. Simultaneously, new content creation devices, such as digital cameras, are embedded. As a consequence, the amount of locally stored content is bound to increase in huge numbers. In order to provide support for end-users in managing this evergrowing pile of content, new means of accessing, organizing, and enjoying the content are needed. We investigate techniques that may be used to display more information, especially visual content, on the mobile device screen at once, as well as accessing the content with ease. We focus on visual interaction, with a media manager as a target application. We present the design factors and a prototype application running on a mobile phone. We show that it is feasible to include spatial cues in the design of mobile user interfaces, and report an initial usability study with very encouraging results.
\end{abstract}

\section{Introduction}

Currently, one of the most common content types in mobile devices is user-created personal content, such as photos, video clips, contacts, and messages. Personal content has some distinctive features: it is often considered important, it is invaluable (in many cases it cannot be replaced), it has emotions attached to it, and it may be very familiar to the user. These characteristics may imply new challenges and possibilities in managing such content.

Content management is a vast research area, including topics such as content transcoding and transfer, data storage technology, searching, security, archiving, and so forth. In this paper, we focus on yet another aspect of content management - the user interface. We concentrate especially on presentation of and interaction with personal content in mobile domain. Our goals are to provide the user with a broad view over the content stored on his/her mobile terminal, and to allow rapid access to any content object.

Displaying arbitrary graphical images on a mobile terminal screen requires addressing several issues. One of these is providing the user with a proper browsing method. The most straightforward method is to display several thumbnail images (heavily diminished versions of the actual images) on the screen at once. The user can then locate the desired image by identifying the contents of the thumbnail.

The restrictions caused by the small screen are evident especially in the browsing task. On a small screen, only a few thumbnail images may be displayed at once. This 
makes browsing a large set of images a tedious task with a lot of scrolling involved. For a presentation point of view, there are two obvious options to address this issue. Either the image thumbnails are made even smaller, so that more of them can be displayed at once, or screen real estate is conserved by the means of distortion. We set out to study yet another approach: carefully applying spatial cues that would allow more efficient usage of screen space, combined with a new interaction technique.

The paper is organized as follows. First, we review the relevant related work, followed by a presentation of our approach and design. We consider the possibilities and limitations of small screen graphics and mobile interaction, and present the design decisions in detail. We then describe the results of a usability study, followed by discussion and conclusions.

\section{Related Research}

\subsection{Photo Browsing}

Several user interface solutions for browsing photos on a large screen have been developed. The most common is inevitably a grid-based layout with thumbnail images, available in Windows XP and virtually every photo application, such as ACDSee [1] and Picasa [12] to name but two. The same approach has been adopted to small screen user interfaces as well, the grid being replaced with a one-dimensional list showing a few thumbnails at a time (Figure 1).

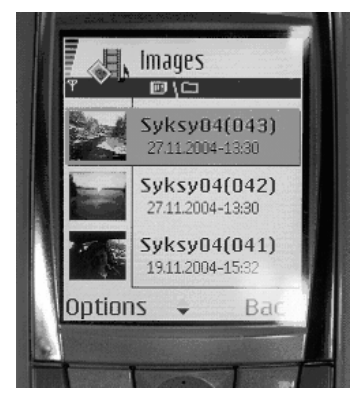

Fig. 1. The Gallery application in current Nokia mobile phones

In addition to thumbnail grids, there are also more advanced browsing user interfaces available. Most photo browsing applications allow the creation of collections. The creation may take place manually, based on, e.g., user-created albums, or automatically, based on, e.g., different categorization techniques. Even though the creation of collections is beyond the scope of this paper, there is one related concept worth noticing: the inclusion of hierarchy, either explicit (e.g., an album) or implicit (e.g., time). What is common to many of these hierarchy-based approaches is dividing the browsing task into two phases:

- the filtering phase and

- the browsing phase. 
This means that in order to have a reasonable amount of objects to browse, the user is first expected to filter the undesired content out of view (to zoom in into the hierarchy), and only then start browsing.

A common approach is to replace the traditional folder-based approach with a temporal view, e.g. [4,5]. As an example, Graham et al. [5] present Calendar Browser, which allows viewing images on a single temporal granularity level at a time, such as a year or a month. A maximum of 25 images are displayed at once; therefore, some summarization algorithms are needed to find representative images when more than 25 are available for a chosen timeframe. On a year level, for example, the images are labeled according to months; when a user clicks on a photo, the browser zooms in into the month the photo was taken. Again, 25 photos from that month are displayed. Selecting a photo on this level further zooms in into the day level. On this level, no summarization is applied, but all photos are browsable with a traditional grid view and Next/Previous buttons.

In addition to time, location is also a natural criterion for organization. One photo browser that takes location into consideration is Nokia Album [2]. It allows clustering the images by both time and location. The location information is retrieved automatically from the GSM network and attached to photos as they are taken.

Beyond Nokia Album, we are not aware of any published research addressing image viewers for mobile phones as such. However, photo browsers for PDAs have been studied earlier. For instance, Pocket PhotoMesa [8] uses treemaps for image layout and zooming for navigation. Image browsing on small screen devices have also been studied by Harada et al. [6]. They compared a folder-based traditional layout with a vertical timeline. Among other things they discovered that the zoomable timeline with system-generated time-based hierarchies was at least as effective for browsing as was the traditional layout.

Lifeblog [10] is another example of a timeline-based content browser and organizer. It runs on both select mobile phones and Windows PCs. In addition to browsing photos, Lifeblog can be used to synchronize personal content between the phone and a PC, including photos, messages and videos. In Lifeblog, the timeline is not hierarchical, but linear.

Lehikoinen and Aaltonen [9] present a distortion-based method for displaying more images on a small screen. A perspective distortion is applied to photos, resulting in more screen space available for additional information, such as a menu (Figure 2). The authors found out that a small amount of distortion did not decrease the time it took to recognize an image.
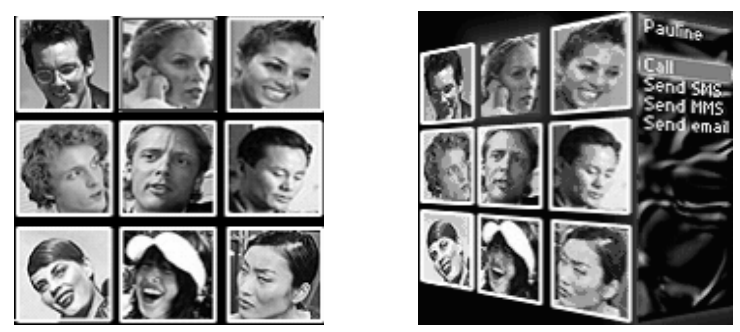

Fig. 2. Perspective distortion frees some screen space [9] 


\subsection{Information Visualization}

Basically there are several techniques for presenting large information spaces in a compressed form. Noik [11] has classified these as follows: implicit (use of perspective), filtered (removal of objects with low degree of interest), distorted (size, shape and position), and adorned (changing attributes such as color).

Many of the current visualization methods suitable for small screens rely on distorting the view; i.e. the viewpoint information is manipulated in a way that enables seeing important objects in detail, while preserving the broader context to which the object belongs. For example, the rubber sheet [13] is a view distortion technique that allows the user to choose areas on the screen to be enlarged. Therefore, the whole information space can be displayed at once with very low amount of detail. Should the user want to see some areas in more detail, he or she stretches the rubber sheet on the particular screen location, effectively zooming into the information on that area.

Zooming and zoomable user interfaces (ZUI), such as PAD++ [3], are another way of presenting large information spaces even on a small screen. The information is presented on a 2D space, and the user can pan the view as well as zoom in and out of any part of that space. The view transition is animated to maintain the broader context of the local detailed information.

Kamba et al. [7] present a way of saving screen space by using pop-up type interface components (the controls are hidden until needed), and movable interaction elements (the elements can be arranged on the screen so that maximum working area is retained).

\section{Our Approach}

There is an inherent paradox in combining visual interaction and limited screen space. Visual interaction necessarily takes up some screen real estate for the controls, leaving less space for the content itself. There are two basic approaches to maximizing the amount of the content displayed on the screen:

- minimizing the interaction widgets (or hiding, making transparent etc. when not needed), or

- compressing the content into a more compact form.

We combined these two approaches by keeping the interaction elements visible but minimizing their visual appearance, and by applying slight spatial cues in order to make more content fit on the screen. As a result, a photo browsing application named MediaTray was developed.

Visualizing any information on a small screen requires very careful crafting. In order to find the optimum techniques and enhancements, one has to create both conceptual still images and animated sequences. Our study makes no exception: we created dozens of still images and a number of different animated versions of the concept until we were satisfied with the result and were able to proceed.

We aimed at developing a content browsing application that would offer a quick and easy way to browse media objects. The application should be able to show more 
images on the screen than what is currently possible. Further, we aimed at finding out whether it is feasible to incorporate any spatial cues into a small screen user interface design.

The application should be usable with the current imaging phones equipped with four arrow keys, a selection key, two software keys for application dependent functionality, and a color screen of $176 \times 208$ pixels.

\subsection{The MediaTray Concept}

An early version of the concept was based on folders. However, with initial heuristic evaluations it became quickly apparent that working with folders is not natural in the mobile domain: people are not familiar with using folders in their mobile terminals, not to mention creating them. On the contrary, it appeared that time is one of the most important aspects for classifying digital photos. This is also reported in e.g. [2]. As a consequence, a temporal organizational approach was adopted.

On a concept level, MediaTray consists of two primary screen components: the time bar and the content area (the tray). The time bar is used to filter and control the time span that is displayed on the tray at a time. In addition to these components, some controls for navigation are needed; they are primarily located at the tray frames in order to save screen space. The final version of the application prototype, running on a mobile phone, is shown in Figure 3.
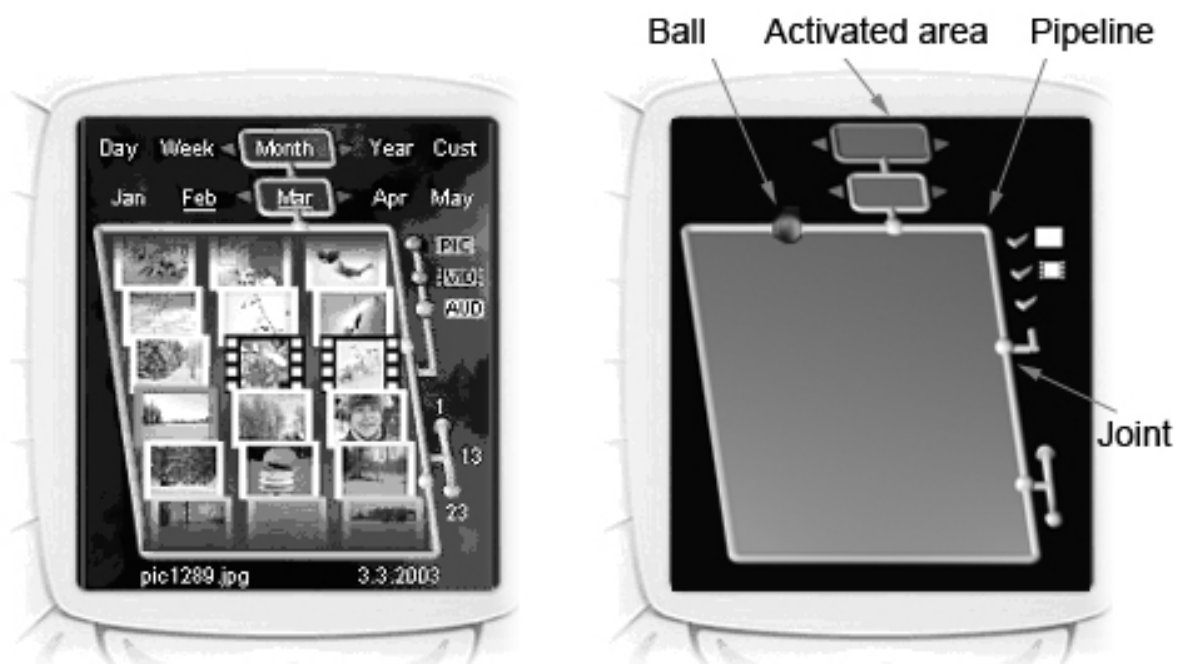

Fig. 3. The MediaTray (on the left) and the user interface components (on the right). The time bar consists of two rows at the top, whereas the tray area fills most of the remaining screen space. The ball is a cursor that is moved along the pipeline.

\subsection{Screen Components Explained}

The time bar consists of two rows of text on the screen (Figure 3 ). The first row is used for selecting the temporal granularity level: it determines the time span length 
within which images on the tray area are displayed. The selection may be based on a day, week, month, or year level. Further, the Cust setting allows creating personalized collections that are not necessarily time-based. It may contain, e.g., pictures of animals only. Rows are horizontally scrollable.

The contents of the second row will change according to the selection made on the first row. For example, if "Month" is selected, the second row shows the names of months (the year will be the one chosen earlier, or this year if no selection has been made). The first and second row selections always remain in the same horizontal position on the screen and are marked with a red frame when focused. The frame will change to blue when the input focus is lost. Underlining is used to indicate that some content exists. For example, in Figure 3 there are some photos taken in February and March, but none in January.

The tray contains the images, filtered according to the time bar settings. The tray is framed; the frame serves also as an input indicator (see the section "Interaction and navigation" below). In addition to the tray area, also the objects are framed. An object's frame indicates its media type. For example, a video clip has a filmstrip-like frame. In this paper, however, only images are considered.

The tray is slightly slanted. This visual design decision serves two purposes: first, it gives a cue of perspective (even though the photos themselves are not distorted), and thus allows the user to organize the thumbnails as if they were very slightly behind each other. Second, the images are easier to differentiate from each other when their borders are not aligned to grid.

We considered several options prior to ending up to slanting. Some of these options are presented in Figure 4 (it is worth noticing that all presented options contain the same amount of images). The leftmost image is the most obvious solution - a grid without any distortion. This takes up a considerable amount of screen space, however. The next option is to decrease the thumbnail spacing (the middle image in Figure 4). This resulted in a crowded-looking, visually unpleasant design where photos seemed to occlude each other without any obvious reason. Finally, we slanted the tray in order to provide a hint of perspective, arranged the images based on a slanted grid, and made them appear very slightly on top of each other.
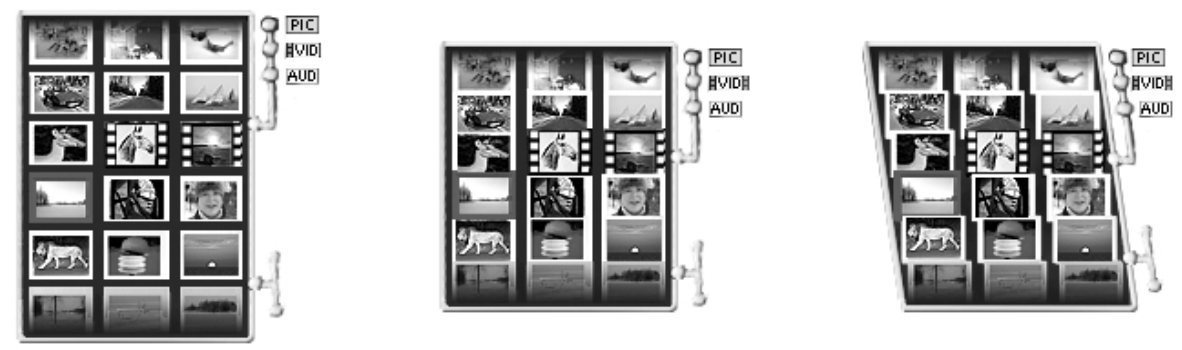

Fig. 4. A comparison of thumbnail presentation options

It is worth noticing that the first folder-based version had a larger preview of the currently selected image. It was left out from the timeline version, since in heuristic and walkthrough evaluations during the concept creation, we found out that the 
images were recognized also without the preview, and that when a user wanted to view the whole image, they wished to see it on full screen regardless of the preview.

\subsection{Interaction and Navigation}

The fundamental design decision concerning interaction and navigation was preventing the user from getting lost. As a consequence, we designed an interaction model where the input focus is always visible, and always presented in a consistent fashion. This is especially crucial in systems based on discrete input with no cursors or pointers. In our prototype the user uses 5-way navigation key for navigating inside the application. Viewing a picture in full screen mode is done by pressing the navigation key.

In order to emphasize the importance of lostness-free navigation even further, we designed visible navigation paths. MediaTray consists of pipes and joints that indicate the possible navigation paths (pipes) and interactive components (joints).

The navigation is based on a "move the ball in the pipes" metaphor. Any interactive component is connected to others via the pipes. This way, the user not only always knows where the focus is, but immediately recognizes the possible directions for navigating further. The ball is animated and moves fast along the pipe system. This helps the user to follow where the selection is going to or coming from.

When wishing to browse photos, the user simply "jumps" from the pipe to the tray area. The tray area is accessible from any direction and the user presses the navigation key to direction where the tray area is located. When doing this, the target picture (the one closest to the joint from which the jump was made) is framed with red. There are three pictures in a row and an infinite number of rows, depending on how many pictures there are in the selection. Six rows are visible at a time. If the selection is larger than six rows, the first and/or last visible rows are dimmed a little. This gives the user a signal that there are more pictures but they are not visible.

When the user wishes to exit the tray area, they simply move left from the leftmost image, or right from the rightmost image. There are no visible joints for doing this, but what we call virtual joints are used. Virtual joints are operative only when moving from tray area back to the pipe. The reason for including virtual joints is that they provide an easy access back to the tray area if the user has accedently exited from there. Otherwise the ball would have moved automatically to the closest joint. This would have been confusing. Furthermore, we did not want to add too many joints along the routes, but to maintain efficiency of movement in the application.

When a picture is selected in the last visible row and the user wants to move further downwards, all visible pictures are scrolled upwards and a new row appears at the bottom of the tray area as the first row disappears. If the amount of browsable images is very high, the user may choose which part of the collection is shown by moving the focus to the scroll lever (on the lower right corner) and adjusting the lever to the desired position. This enables the user to go to the beginning or the end of a large selection very fast. The current position is displayed next to the lever, and the size of the collection below it.

On the upper right corner of the tray area there is a bar for selecting which media types are shown. In the current version there are three possible choices available: picture, video, and audio. This is a multi-select control with at least one selection on all the time. A selected item is marked with green. 


\subsection{On the Graphical Model}

The MediaTray application is based on a pure 3D model (Figure 5). Even though the model is pure 3D, we do not allow free camera nor object manipulation. This is due to practical reasons: the current input capabilities would pose rather huge challenges for easy interaction with a six degrees of freedom manipulation. Therefore, the object manipulation always takes place in two dimensions. The third dimension, in this case depth, is present but is used as a visual cue and aid for cognitively determining an object's location related to others.

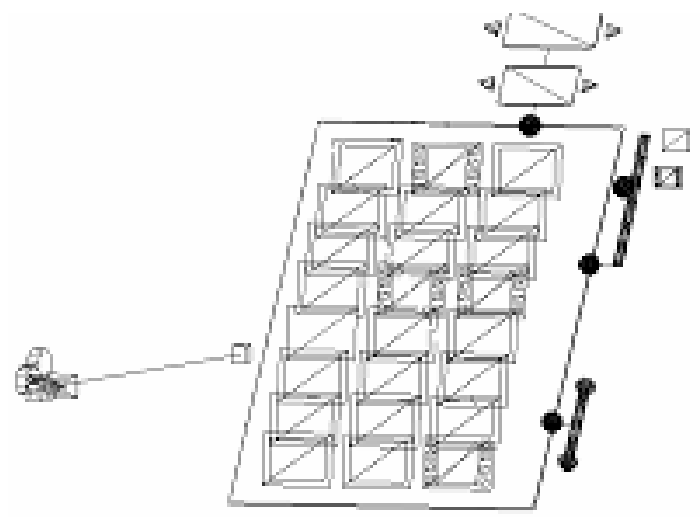

Fig. 5. The graphical model

The object constructions are made by special 3D modeling and rendering software (3DSMax). Only by using this kind of method it is possible to find the best views and appearances of the object; the object orientation is carefully taken into account.

There is a slight shadow effect behind each media object, which gives a feeling of more depth. Also, a blue gradient slide in the first and last row gives a feeling of continuation of the objects; when the gradient is not visible there are no more objects behind.

Using a camera inside the 3D rendering software gives a lot of possibilities to see the objects in a different way. We tried distorted, more slanted, wide-angle views and many other ways to find the best model construction. The conclusion was simple: no distortion in the structure, all media objects of the same size, pipeline always visible (not covered by any objects) and one red spot or frame indicating the selection.

The background of the application turned out to be important. We tried different configurations in early stages and come up with conclusion that it is dark enough to give more contrast, it is fuzzy to give a feeling of floating objects and it can also be slightly animated to give a feeling of non-static environment.

\section{Evaluation}

In order to assess the usability of the concept, we arranged a qualitative evaluation. The purpose of the evaluation was to find out how easily users could find, browse and view images with the application, and what they subjectively thought about the design. 


\subsection{Participants}

Nine persons, including one pilot user, participated in the evaluation. Their median age was 33 years. Out of the nine participants two were female. All participants had technical background and a university degree. None of the participants has any prior experience with MediaTray and they all volunteered in the evaluation. Each was rewarded with a small gift.

The criterion for the participants was that they should have taken photos with a camera phone. The activity level in taking photos varied between the participants. They had also different ways to store the images. Some participants stored them in the mobile phone, while some transferred them to the computer every now and then. At the time of the usability test, the number of images stored in a mobile phone varied from 0-5 to 20-30. Three participants had created folders for the photos, such as "Work", "Holiday trip" or "Old pictures". Others had left them as a single list.

\subsection{Apparatus}

The tests were carried out with a prototype application installed in a Nokia 3650 mobile phone (Figure 6). A fixed set of images was used.

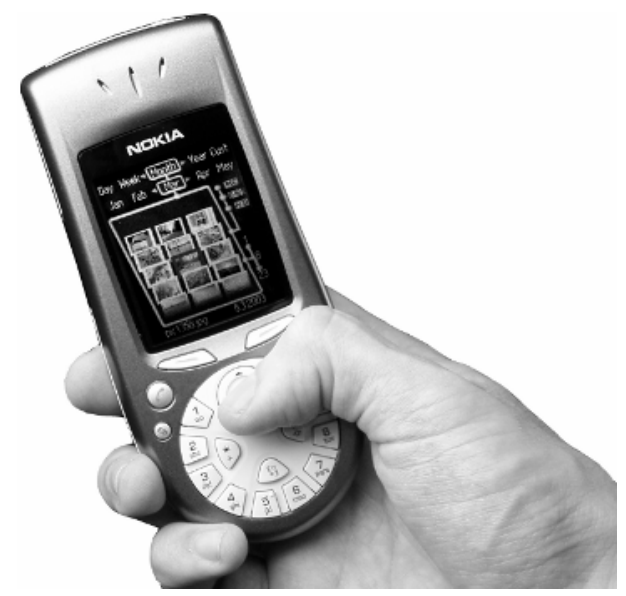

Fig. 6. MediaTray application running on a Nokia 3650

\subsection{Procedure}

There were nine basic tasks in the test. The tasks dealt with browsing and viewing photos, selecting a correct date in the time bar and defining visible file types.

The tests were arranged in a laboratory environment. There were one participant and the moderator present at a time. In the beginning, the test participant was familiarized with the test equipment and procedure. They were also interviewed about their demographics. The participant tried independently navigation and picture selection prior to actual tasks. After the warm-up, the participants were asked to conduct test tasks one by one, and think aloud while proceeding. Finally, there was a discussion about the design. 


\section{Results}

\subsection{Tray Area}

Our first goal was to display more images on the screen. There was a maximum of 18 thumbnails visible on the tray area, which is remarkably more than normally. Participants were satisfied with that, considering the screen size. None of them commented that slanting the tray would have had any effect on thumbnails. However, shrinking the size of thumbnails had some drawbacks in recognizing them, but that was foreseeable. In some cases thumbnails were considered to be blurry and a little too small, but participants said recognizing images would be easier if they would have taken these photos by themselves. It should be noted that this is only a user comment, not a verified result.

Selecting an image was done mainly based on a small thumbnail. Date taken and the image name were visible at the bottom of the screen, but participants did not use them very often or at least they did not mention that they would have looked at them.

In our design, first and last rows were dimmed in order to indicate that there were more images available either above or below visible images. Participants thought that this was quite an evident method and none of them had any problems in checking all images from the selection.

\subsection{The Time Bar}

The Time bar is a very crucial part of our user interface since users usually need to filter images to find the ones they are looking for. Participants learned very quickly how to use this control and they found the correct view almost instantly. However, the time bar was not utilized to its full extent: participants accomplished tasks mostly in a month view. Other views were used only if absolutely necessary or specified explicitly in the task description. Participants said that the weekly view is not very important because it is quite a short period, and events usually start during weekend and continue the following week whereas in calendar view the week normally starts on Monday. The day view was used only if the participant needed to check a certain date and look for an image from that day. Year was considered to be too long a period: the large amount of images would make browsing time-consuming.

Dividing the time bar into two halves had both advantages and disadvantages. Participants could easily browse e.g. days or months on the second row once they had selected a view from the first row. Furthermore, the time bar also indicated if there were no images a specific day or month and thus allowed bypassing empty slots quickly. This feature proved to be useful.

Problems were related to selecting the right view; sometimes participants had difficulties in doing that. All broader scale selections in the time bar affected the detailed views and adjusted values on the second row accordingly. For example, the selected month defines available dates in the day view. The current year was indicated at the bottom of the screen, but apparently participants did not notice that until they had tried to look for images taken on a wrong year. 


\subsection{Navigation}

Our second goal was to offer a good and error-free navigation in browsing media objects. The pipeline structure and a red ball as a cursor seemed to work well for this purpose. Participants were quite enthusiastic about the idea of the pipeline structure used for directing cursor movements and some of them found navigation in the application also entertaining and fun. They said that the cursor movements on the pipeline reminded them from some video games from the 80's and some tasks almost turned into a gaming session as participants "drove" around the pipeline.

In addition to visible joints, there were also some virtual joints for specific purposes. In order to provide a smooth exit from the tray back to the pipeline, the participants could just move the cursor from the outermost image to the direction of the pipeline; the cursor jumped on the pipe and stayed alongside the image. From that point it was possible to move to another part of the application, time bar, or return back to the tray area. During the test some participants exited unintentionally from the tray area because they thought that the focus would move automatically to the next row if they press the joystick right on the rightmost image as it would happen in the terminal's menu structure. However, as the cursor just moved to the pipeline but did not move anywhere from there, they could easily return and continue browsing images. Participants noted this behavior very quickly and they did not make this error anymore once they had noticed it.

Transition between the tray area and the pipeline was not completely seamless. Even though it was possible to exit from any outermost media object back to the pipeline, it did not always work the other way around, especially with a small number of images on the tray. For example, from the scroll bar joint it was not possible to move into the tray if there were so few images that the row aligned with the scrollbar joint was empty.

Another problem was related to navigation at the top of the tray area. It was not possible to move directly to the time bar from virtual junctions, but the user had to move first to the right. Some participants tried upward navigation because they thought it would automatically take them to the time bar.

In overall participants learned very quickly how to navigate in the application even if some directions were not so obvious. For instance, navigating down at the scrollbar joint will "drive" the cursor to the time bar joint at the top of the screen because there are no fixed joints at the bottom of the screen or on the left side of the tray area. On the other hand this worked as a shortcut to the time bar almost everywhere from the tray area and hence, it was adopted.

\section{Discussion and Future Work}

The results from the test were very encouraging. The design goals that we set at the beginning were well met and the participants were satisfied with how the application worked. However, there are some things that could be improved in subsequent versions.

Increasing the number of images on the tray area will evidently have an affect on the size of the thumbnails and thus make recognizing them more difficult. 
Furthermore, we did not show image names at all since they are often arbitrary. The creation date was visible, though. It remains to be seen how much familiarity with the photos helps in recognizing small thumbnails and finding the right image. In the posttest interview participants were confident that they could recognize images more accurately if they would have taken them themselves.

Another thing is recognizing different media types on the tray area. In our design we sketched that other media types, like video and audio, could also be browsed in this application. Especially presenting audio files on the tray area is a challenging task because we did not show file names or other textual information, which is traditionally used for identifying audio clips. Videos could be presented in a similar way as images, but the border around a thumbnail (a frame from the video) could be a filmstrip to indicate the media type. For audio files a thumbnail could be some graphical metadata information, an album cover or a picture of an artist. However, such as audio files should have their own border style as well in order to distinguish them from other media types. One possibility is to extend the preview capability of the application required by different media types. The application could play a short video clip or audio sample when a user browses the objects and pauses on one.

The time bar worked well and participants were able to select the correct view smoothly. The visibility of selected views should be improved because some participants had problems, if only minor ones, in selecting a correct view. Most importantly, all selected values should be clearly visible near time bar. This would improve awareness of selections. Also the order of views could be rethought. Currently the time bar has been organized from the most detailed view (day) to the broadest view (year). However, the selection of images on the tray area is first defined by the year and the user can narrow the selection into a more detailed view if necessary.

Based on the results from the usability test, basic navigation in the pipeline structure was very intuitive and easy even though it was new to participants. All problems in navigation were related to virtual joints. Participants did not know for sure how they worked and what were the available navigation directions. Therefore, virtual joints require some more detailed interaction design on how they should work in each case and whether there are any new locations that would require a virtual joint.

One of the key findings was that we are actually able to decrease the icon spacing without losing attractiveness, when some slight spatial cues are applied. The slanted tray enabled more images to be placed on the screen, which is exactly what participants wanted to have. The appearance of the application was also appreciated. It looked different compared to normal applications in the phone, but in a positive way.

\section{Conclusions}

We have developed a mobile application prototype for photo browsing on a small screen. Our design goal was to provide an application that allows a quick and easy access to all media content on a terminal. Our approach is based on visual interaction; we developed user interface widgets that we call pipes and joints. The design allows the user to move the selection tool, a ball, rapidly through the pipe system. The ball stops whenever there is a joint in the pipe. A joint is an active component where some interaction may take place. The benefits of the piping system are visible navigation paths and the fact that the user never loses sight of the currently focused object. 
Another aspect of the UI design is applying slight spatial cues. In this case, we tilted the object plane and arranged objects so that they seemed to appear in threedimensional space. As a consequence, we were able to arrange the icons representing the objects more efficiently than would have otherwise been possible.

In order to assess usability of the concept, we arranged an evaluation. The results showed that our concept is very easy and even fun to use. One of the key findings was that we are actually able to decrease the icon spacing without losing attractiveness, when some slight spatial cues are applied.

\section{References}

1. ACDSee. Online document, availabe at http://www.acdsystems.com/English/index.htm. Last checked Apr 25, 2005.

2. Ahtinen A., Andersson M. Learnability and Automatic Clustering of Images in Mobile Devices. In Proceedings of MobileHCI 2004, pp. 404-408.

3. Bederson B.B, Hollan J.D. Pad++: A Zooming Graphical Interface for Exploring Alternate Interface Physics. In Proceedings of the ACM UIST'94 Symposium on User Interface Software and Technology. 1994. pp. 17-26.

4. Cooper M., Foote J., Girgensohn A., Wilcox L. Temporal event clustering for digital photo collections. In Proceedings of the eleventh ACM International Conference on Multimedia, 2003, pp. 364-373.

5. Graham A., Garcia-Molina H., Paepcke A., Winograd T. Time as essence for photo browsing through personal digital libraries. In Proceedings of the second ACM/IEEE-CS joint conference on Digital Libraries, pp. 326-335.

6. Harada S., Naaman M., Song Y.J., Wang Q.Y., Paepcke A. Lost in memories: interacting with photo collections on PDAs. Proceedings of the 4th ACM/IEEE-CS joint conference on Digital libraries, 2004, pp. 325-333.

7. Kamba T., Elson S.A., Harpold T., Stamper T., Sukaviriya P. Using Small Screen Space More Efficiently. In Proceedings of CHI 1996, ACM, pp. 383-390.

8. Khella A., Bederson B.B. Pocket PhotoMesa: A Zoomable Image Browser for PDAs. Proceedings of the 3rd international conference on Mobile and ubiquitous multimedia, 2004, ACM, pp. 19-24.

9. Lehikoinen J., Aaltonen A. Saving Space by Perspective Distortion When Browsing Images on a Small Screen. In Proceedings of Australasian Computer Human Interaction Conference (OZCHI) 2003, pp. 216-219.

10. Lifeblog. Online document, available at http://www.nokia.com/nokia/0,1522,,00.html? orig=/ lifeblog Last checked Apr 25, 2005.

11. Noik, E. A Space of Presentation Emphasis Techniques for Visualizing Graphs. In Proceedings of Graphics Interface 1994, pp. 225-233.

12. Picasa Photo Organizer. Online document, available at http://www.picasa.com/picasa/. Last checked Apr 25, 2005.

13. Sarkar M., Snibbe S.S., Tversky O.J., Reiss S.P. Stretching the Rubber Sheet: A Metaphor for Viewing Large Layouts on Small Screens. In Proceedings of UIST 1993, ACM, pp. 81-91. 